drug targets ${ }^{9}$. Inhibition of Vif-APOBEC3G interactions has been shown to restrict HIV infection through the mutation and degradation of uracil-containing viral DNA. Targeting Vif-APOBEC3G interactions may have additional therapeutic benefits by rendering HIV-infected cells sensitive to NK cell-mediated lysis ${ }^{2}$. Combining the administration of small molecules that interfere with Vif with simultaneous NK cell-activation protocols or infusion of NK cells may further potentiate the antiviral effects of the small molecules. The administration of type I interferons, which enhance NK cell activity, may have the added benefit of increasing APOBEC3G expression and inducing other antiviral activities. However, studies have also demonstrated that partial inhibition of Vif can lead to drug-resistant HIV strains due to hypermutation ${ }^{10}$. Furthermore, chronic infection with HIV is associated with a specific defect in NKG2D-mediated activation of NK cells due to lower expression of NKG2D ${ }^{11}$. This lower NKG2D expression is associated with higher concentrations of MICA, the soluble form of the NKG2D-L, in serum from HIV-infected patients. Thus, HIV may indirectly suppress the recognition of HIV-infected CD4 ${ }^{+} \mathrm{T}$ cells by NK cells by enhancing the secretion of NKG2D-L into the serum, which results in profound impairment of NK cell function. These factors will have to be considered in the design of Vif antagonists.
COMPETING FINANCIAL INTERESTS

The authors declare no competing financial interests.

1. Altfeld, M., Fadda, L., Frleta, D. \& Bhardwaj, N. Nat Rev. Immunol. 11, 176-186 (2011).

2. Norman, J.M. et al. Nat. Immunol. 12, 975-983 (2011).

3. Planelles, V. \& Barker, E. Mol. Aspects Med. 31 398-406 (2010).

4. Cerboni, C. et al. J. Gen. Virol. 88, 242-250 (2007).

5. Chiu, Y.-L. \& Greene, W.C. Annu. Rev. Immunol. 26 317-353 (2008).

6. Ramiro, A.R. et al. Nature 440, 105-109 (2006).

7. Gasser, S., Orsulic, S., Brown, E.J. \& Raulet, D.H Nature 436, 1186-1190 (2005).

8. Langevin, C. et al. J. Virol. 83, 10256-10263 (2009).

9. Harris, R.S. Nat. Biotechnol. 26, 1089-1090 (2008).

10. Mulder, L.C.F., Harari, A. \& Simon, V. Proc. Natl. Acad. Sci. USA 105, 5501-5506 (2008).

11. Nolting, A. et al. Virology 406, 12-20 (2010).

\title{
Peli1 (rel)ieves autoimmunity
}

\author{
Paul N Moynagh
}

\section{T cell tolerance is essential to the prevention of autoimmunity. The ubiquitin E3 ligase Peli1 acts as a negative regulator of $\mathrm{T}$ cell activation and contributes to the maintenance of self-tolerance.}

\begin{abstract}
The immune system has an amazing capacity to discriminate between self and non-self while also being able to recognize a bewildering array of foreign chemical entities. This is made possible by the diverse receptor repertoire of lymphocytes. In the thymus, T cells that express high-affinity receptors for self antigens are eliminated. Some T cells, which express low-affinity receptors for self antigens or with receptors for antigens that do not reach the thymus for presentation, escape thymic deletion and are released into the periphery. A critical peripheral tolerance mechanism that prevents these self-reactive T cells from triggering autoimmune responses includes the induction of a state of unresponsiveness known as ' $\mathrm{T}$ cell anergy' ${ }^{1}$. In this issue of Nature Immunology, Chang et al. identify the ubiquitin E3 ligase Pelil as a key negative regulator of $\mathrm{T}$ cell activation, with deficiency in Pelil being associated with loss of self-tolerance and the manifestation of spontaneous autoimmunity ${ }^{2}$.

Full activation of a $\mathrm{T}$ cell requires two signals. The first signal is provided by the $\mathrm{T}$ cell antigen receptor (TCR), and the second is provided by a costimulatory receptor such as CD28. Activation of the transcription factor
\end{abstract}

Paul N. Moynagh is with the Institute of

Immunology, National University of Ireland

Maynooth, Maynooth, Ireland.

e-mail: paul.moynagh@nuim.ie
$\mathrm{NF}-\kappa \mathrm{B}$ is one of the most critical outcomes of this costimulation ${ }^{3}$. Lack of NF- $\kappa \mathrm{B}$ is associated with immune deficiencies, whereas abnormal activation of NF- $\kappa B$ is associated with autoimmunity and cancer. The NF- $\kappa$ B family consists of five Rel proteins that can form dimers in various combinations to regulate gene transcription. In most resting cells, dimeric NF- $\kappa \mathrm{B}$ complexes are sequestered in the cytoplasm by inhibitory I $\mathrm{I} B$ proteins. Innate immune receptor systems such as Toll-like receptors (TLRs) engage adaptor proteins such as MyD88 and TRIF to trigger activation of an I $\mathrm{B}$ kinase complex that catalyzes the phosphorylation of $\mathrm{I} \kappa \mathrm{B}$ proteins, thus promoting their degradation by the proteasome. Liberated NF- $\kappa \mathrm{B}$ translocates to the nucleus and drives the transcription of genes that encode proinflammatory molecules. In $\mathrm{T}$ cells, the simultaneous signaling of TCR and CD28 also strongly stimulates the I $\mathrm{BB}$ kinase complex, activates NF- $\mathrm{BB}$ and induces CD28-responsive genes such as those encoding interferon- $\gamma$ and interleukin 2 (IL-2), which drive the activation and proliferation of $\mathrm{T}_{\text {cells }}{ }^{3}$ (Fig. 1). Robust costimulation by TCRCD28 leads to strong T cell activation, whereas engagement of the TCR in the absence of CD28 costimulation results in T cell anergy.

Chang et al. now identify Peli1 as a mediator of the anergic process that acts as a negative regulator of TCR-CD28 signaling ${ }^{2}$. CD4 $4^{+}$and $\mathrm{CD}^{+} \mathrm{T}$ cells from Peli1-deficient $\left(\right.$ Peli1 $\left.^{-/-}\right)$ mice show hyperactivation and greater proliferative responses to TCR-CD28 signaling. These cells are also less susceptible to anergy and suppression by regulatory $\mathrm{T}$ cells ( $\mathrm{T}_{\text {reg }}$ cells). Aging Peli1 $^{-/-}$mice also have a greater frequency and number of memory $\mathrm{T}$ cells but a lower frequency of naive cells. These mice have enlarged peripheral lymph nodes and substantial tissue infiltration by cells of the immune response. Peli1 $^{-/-}$mice show distinct signs of spontaneous autoimmunity with age, as demonstrated by the detection of serum antinuclear autoantibodies and prominent deposition of immune complexes in the kidney. Furthermore, T cells from $\mathrm{Peli1}^{-/-}$mice show more pathogenic potential in experimental autoimmune encephalomyelitis, an inducible autoimmune model of multiple sclerosis. All these findings suggest a $\mathrm{T}$ cell-intrinsic role for Peli1 in negatively regulating $\mathrm{T}$ cell signaling, facilitating selftolerance and protecting against autoimmunity. An elegant mechanistic explanation for these effects is provided by the identification of c-Rel as a Peli1 target ${ }^{2}$. Signaling by TCR and CD28 together induces Pelil expression, which leads to its interaction with and polyubiquitination of c-Rel, resulting in degradation of the latter (Fig. 1). Loss of Peli1 thus leads to late-phase hyperactivation and nuclear accumulation of c-Rel in response to TCR-CD28 signaling and given the role of $\mathrm{c}$-Rel in promoting the activation, proliferation and differentiation of 


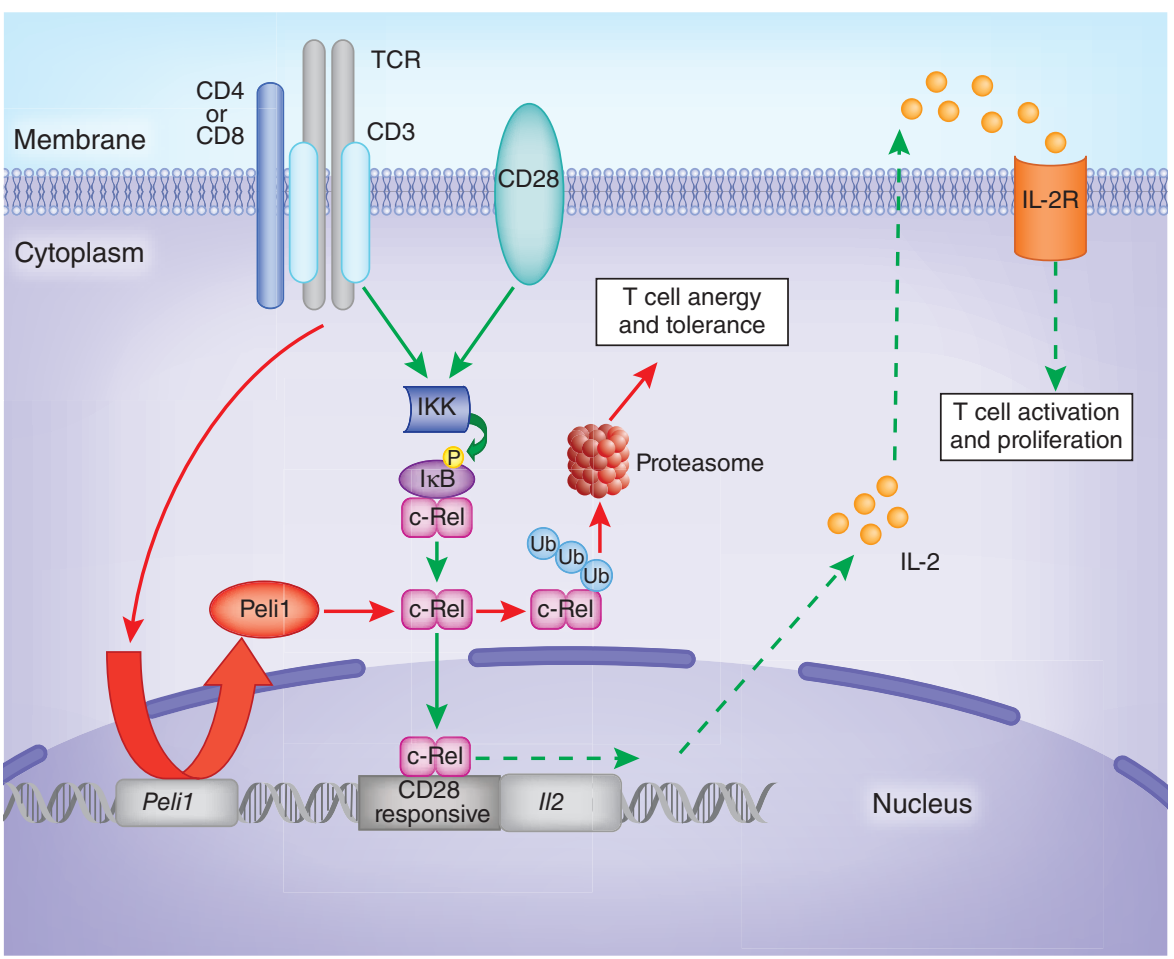

Figure 1 Peli1 negatively regulates T cell signaling by targeting c-Rel. Costimulation of CD28 and the $\mathrm{CD} 3$ subunit of the TCR complex in $\mathrm{CD}^{+}$or $\mathrm{CD}^{+} \mathrm{T}$ cells triggers a complex series of membrane proximal signaling events that culminate in activation of the I KB kinase (IKK) complex. This complex subsequently phosphorylates $\mid \kappa B$, leading to its degradation and the release of Rel subunits of the $\mathrm{NF}-\kappa \mathrm{B}$ family. It is also possible that Rel subunits may be activated by I $\mathrm{kB}$ kinase-independent mechanisms ${ }^{3}$. Free c-Rel translocates to the nucleus and binds to the CD28-responsive region of genes such as $1 / 2$, leading to more transcription. IL-2 is a mitogenic factor for T cells and promotes T cell proliferation. The TCR-CD28 pathway can also induce expression of Peli1 by an uncharacterized pathway. Peli1 interacts with c-Rel and catalyzes its polyubiquitination (Ub), which results in degradation of c-Rel by the proteasome. This inhibits the induction of cytokines such as IL-2 and results in a state of unresponsiveness known as ' $T$ cell anergy' that is associated with peripheral T cell tolerance. IL-2R, receptor for IL-2.

T cells, this is a very plausible basis for the loss of self-tolerance and spontaneous autoimmunity in Peli1-deficient mice.

It is especially interesting that the regulatory role of Peli1 shows some degree of cell specificity, in that the effects of Pelil deficiency are greater in $\mathrm{CD}^{+} \mathrm{T}$ cells than in $\mathrm{CD} 4^{+} \mathrm{T}$ cells ${ }^{2}$. All nucleated cells can express major histocompatibility complex class I and present antigen to $\mathrm{CD}^{+} \mathrm{T}$ cells, so the potential for activation of self-reactive $\mathrm{CD} 8^{+} \mathrm{T}$ cells is especially high. It is reassuring from a self-tolerance perspective that these cells have a strong regulator such as Pelil that can temper cell activation. Given the spontaneous autoimmunity phenotype of Peli1-deficient mice, it is perhaps surprising that these mice have slightly more $\mathrm{T}_{\text {reg }}$ cells in the thymus and periphery. However, the Peli1c-Rel relationship may provide an explanation, as c-Rel acts an important driver of the thymic development of $\mathrm{T}_{\text {reg }}$ cells by directly promoting transcription of the gene encoding the signature Treg cell transcription factor Foxp3 (ref. 4). Paradoxically, Peli1 may negatively regulate the development of $\mathrm{T}_{\text {reg }}$ cells while facilitating the suppressor effects of Treg cells and its effector cytokine TGF- $\beta$ on $\mathrm{T}$ cell activation. The role of Peli1 in the latter is probably dominant, given the spontaneous autoimmunity that is apparent in Peli1 ${ }^{-/-}$mice $^{2}$. It is also intriguing that the negative effects of Peli1 on TCR-CD28 signaling seem to be confined to peripheral T cells. Although strong TCR-CD28 signaling is required for the activation of $\mathrm{T}$ cells in the periphery, one of the key central tolerance mechanisms used in the thymus is the deletion of autoreactive $\mathrm{T}$ cells by self antigen-induced TCR-CD28 signaling ${ }^{1}$. It might be possible that loss of Peli1 may also lead to stronger TCRCD28 signaling in self-reactive cells that might normally escape thymic deletion because of weak activation by low-affinity antigens. Such a scenario would lead to more efficient deletion of autoreactive T cells in the thymus and diminish the likelihood of autoimmunity. However, Peli1 $^{-/-}$mice develop spontaneous autoimmunity instead, which again emphasizes the prominent role of Peli1 in peripheral T cells.
The Chang et al. study also highlights key differences in the function of Peli proteins in the innate and adaptive immune systems ${ }^{2}$. Although Peli1 is able to promote polyubiquitination and degradation of a target protein in $\mathrm{T}$ cells, published studies of Peli proteins have focused on their function in the TLRIL-1 pathways in the innate immune system and have highlighted their tendency to catalyze polyubiquitination of signaling molecules such as IRAK and RIP1, leading to regulation of protein function rather than degradation ${ }^{5,6}$. This raises the question of how Peli proteins can be regulated in different ways and directed toward the catalysis of different types of polyubiquitination with various functional outcomes. In TLR pathways, the E3 ligase activity of Peli proteins can be increased by various kinases $^{7,8}$, and it will be interesting to determine if the activity of Pelil is subject to regulation by TCR-CD28 signaling. Furthermore, Peli1 seems to be able to manifest contrasting effects in different cell settings. Thus, Peli1 negatively regulates the activation of $\mathrm{c}-\mathrm{Rel}$ by TCR-CD28 (ref. 2), whereas it mediates TRIFdependent activation of NF- $\kappa B$ in some TLR pathways by acting as an E3 ubiquitin ligase for RIP1 (ref. 6). The latter function of Peli1 translates into an important role in mediating the TLR3-induced activation of B cells. This raises the fundamental question of why the same molecule would be used to negatively regulate $\mathrm{T}$ cell activation but strongly activate $\mathrm{B}$ cells and how such opposing functions are controlled. Further hints of the dichotomy of Pelil function in the innate and adaptive systems also emerge from its different roles in mediating responses to TGF- $\beta$. Thus, Peli1 facilitates TGF- $\beta$-mediated suppression of T cell activation ${ }^{2}$, whereas TGF- $\beta$ represses inflammatory IL-1 signaling by using the signal transducer Smad6 to inhibit the interaction of Peli1 with IRAK1 in the IL-1 pathway 9 However, the importance of Pelil as a target of TGF- $\beta$ in the IL- 1 pathway is equivocal, given that cells from Peli1-deficient mice have an intact IL-1 signaling pathway ${ }^{6}$.

The study presented in this issue ${ }^{2}$ adds Peli1 to a growing number of E3 ligases that regulate T cell tolerance. Cbl-b and Itch are E3 ligases that, like Peli1, negatively regulate T cell activation and promote tolerance ${ }^{10}$. Whereas Peli1 inhibits c-Rel, a late downstream mediator of $\mathrm{T}$ cell signaling, Cbl-b and Itch target molecules that are more proximal to the TCR-CD28 signaling complex. Such tight regulation of $\mathrm{T}$ cell activation at various levels of the signaling pathway is presumably an attempted fail-safe against loss of self-tolerance and autoimmune disease. E3 ubiquitin ligases are emerging to the fore in this process, and the 
generation of knockout models will aid in determining whether the other Peli proteins, Peli2 and Peli3, regulate $\mathrm{T}$ cell tolerance. Interestingly, Peli2 interacts with Bcl-10, a key intermediate signaling molecule in the TCRCD28 pathway ${ }^{11}$.

Finally, the findings of Chang et al. ${ }^{2}$ justify population studies to explore the role of Pelil in human autoimmune disease. Any Peli1 mutations that affect its function and/or expression will probably to lead to autoimmunity. Furthermore, given that Peli1 effects c-Rel degradation and c-Rel is associated with lymphoid malignancies ${ }^{3}$, it will be very interesting to determine if the Peli proteins are also involved in the cancer arena. In summary, the study by Chang et al. indicates Peli1 is a crucial regulator of $\mathrm{T}$ cell activation and autoimmunity ${ }^{2}$, which represents an exciting discovery that adds to the molecular understanding of the regulatory mechanisms that underlie self-tolerance.

\section{COMPETING FINANCIAL INTERESTS}

The author declares no competing financial interests.

1. Goodnow, C.C., Sprent, J., Fazekas de St Groth, B. \& Vinuesa, C.G. Nature 435, 590-597 (2005).
2. Chang, M. et al. Nat. Immunol. 12, 1002-1009 (2011).

3. Vallabhapurapu, S. \& Karin, M. Annu. Rev. Immunol. 27, 693-733 (2009).

4. Long, M., Park, S.G., Strickland, I., Hayden, M.S. \& Ghosh, S. Immunity 31, 921-931 (2009).

5. Moynagh, P.N. Trends Immunol. 30, 33-42 (2009).

6. Chang, M., Jin, W. \& Sun, S.C. Nat. Immunol. 10, 1089-1095 (2009).

7. Smith, H. et al. Proc. Natl. Acad. Sci. USA 106 4584-4590 (2009).

8. Smith, H. et al. Biochem. J. 434, 537-548 (2009).

9. Choi, K.C. et al. Nat. Immunol. 7, 1057-1065 (2006). 10. Venuprasad, K. Cancer Res. 70, 3009-3012 (2011).

11. Liu, Y. et al. J. Biol. Chem. 279, 37436-37444 (2004).

\section{STING-dependent signaling}

\section{Glen N Barber}

\section{The sensing of pathogen-associated DNA in the cytoplasm is an important trigger of host-defense responses that include the production of type I interferon. A new study suggests that the DExDc helicase DDX41 may function in dendritic cells as a DNA sensor to activate STING-dependent innate immune responses.}

\begin{abstract}
The innate immune system has evolved a series of cellular sensors and signaling pathways that activate host-defense mechanisms in response to microbial invasion ${ }^{1}$. However, how cells detect pathogens that contain doublestranded DNA (dsDNA), such as many types of cancer-causing viruses, intracellular bacteria and perhaps parasites, remains to be resolved. In this report, Zhang et al. identify the DExDc helicase family member DDX41 as a sensor of cytosolic DNA that can trigger the production of host antipathogen genes such as those that encode type I interferons in myeloid dendritic cells (DCs) ${ }^{2}$. The finding has potentially important implications for the understanding of innate immune processes, microbe-based pathogenesis and even autoimmune disease.

Interestingly, DDX41 is not the first DExDc helicase to be linked to the sensing microbial invasion and rapid facilitation of appropriate host-defense countermeasures. For example, the RNA helicases RIG-I, Mda5 and LGP2 are critically important for the detection of invading RNA viruses in almost all cells and for triggering the production of antiviral proteins ${ }^{3}$. The RIG-I family and DDX41 are cytosolic $\mathrm{DExD} / \mathrm{H}$ proteins that directly associate with pathogen-derived nucleic acid released into the cytosol. RIG-I recognizes 5'-triphosphorylated double-stranded RNA (dsRNA), which is
\end{abstract}

Glen N. Barber is in the Department of

Cell Biology, University of Miami School of

Medicine, Miami, Florida, USA.

e-mail: gbarber@med.miami.edu commonly produced by negative-stranded RNA viruses, whereas Mda5 recognizes larger, high-molecular-weight dsRNA species typically encoded by positive-stranded viruses ${ }^{4}$. The interaction of viral RNA with RIG-I and Mda5 induces a conformational change that enables interaction with the mitochondrial adaptor IPS-1 (also known as MAVS, CARDIF or VISA), which facilitates the activation of transcription factors (such as IRF3, IRF7 and NF- $\kappa$ B) that are responsible for inducing the transcription of many genes encoding molecules important for host defense, including type I interferon ${ }^{1,5}$. Zhang et al. show that DDX41 interacts with dsDNA species, including the genomes of DNA viruses such as vaccinia virus and herpes simplex virus type 1 (HSV-1) $)^{2}$. This event, described in myeloid DCs, similarly leads to the production of type I interferon via activation of IRF3 and NF- $\kappa B$ (Fig. 1), although it requires the assistance of the transmembrane-containing, endoplasmic reticulum-associated adaptor STING ('stimulator of interferon genes') ${ }^{6}$. STING has been shown to be essential for the production of type I interferon by cytosolic dsDNA in nearly all cells examined, including mouse embryonic fibroblasts, macrophages and DCs. Accordingly, loss of STING prevents the production of type I interferon in response to transfected plasmids, DNA viruses and certain intracellular bacteria, such as Listeria monocytogenes and possibly even parasites. Mice that lack STING are viable but extremely sensitive to lethal infection with HSV-1. Thus, the STING-regulated pathway is extremely important for host defense against DNAbased microbes.

There are approximately 59 members of the $\mathrm{DExD} / \mathrm{H}$ family; Zhang et al. identify DDX41 by means of an RNA-mediated interference screen to individually suppress the expression of each of these helicases in cells ${ }^{2}$. They then assay the ability of such cells to produce type I interferon in response to cytosolic B-form DNA (poly $(\mathrm{dA}: \mathrm{dT})$ ). They find that the suppression of DDX41 expression in DCs and monocytes impedes the transcription of type I interferons and cytokines such as interleukin 6 and tumor necrosis factor by dsDNA and HSV-1, similar to the loss of STING. HSV-1 replication is concomitantly greater in cells in which DDX41 is suppressed. Loss of DDX41, however, does not affect dsRNA-dependent production of type I interferons, which indicates specificity for the cytosolic DNA-dependent innate signaling pathway. Loss of DDX41 also does not affect the production of interleukin- $1 \beta$ by cytosolic DNA, which indicates that this helicase acts independently of the pathway for the production of proinflammatory cytokines dependent on the cytoplasmic DNA receptor AIM2 and mediated by caspase-1 (ref. 7).

As additional experiments by this group indicate that the DEADc box of DDX41 can bind dsDNA (Z-form and B-form) but not synthetic ssRNA such as $\operatorname{poly}(\mathrm{U})^{2}$, these data collectively suggest that DDX41 associates with dsDNA species from pathogens and subsequently recruits STING for the activation of type I interferons. Published 